5th International Science Congress \& Exhibition APMAS2015, Lykia, Oludeniz, April 16-19, 2015

\title{
On Gibbs Measures of the Potts Model with Three Competing Interactions on Cayley Tree of Order 3
}

\author{
H. AKIN ${ }^{a, *}$ AND H. SAYGILI ${ }^{b}$ \\ ${ }^{a}$ Zirve University, Faculty of Education, Department of Mathematics, Gaziantep, 27260, Turkey \\ ${ }^{b}$ Gaziantep University, Science and Art Faculty, Department of Mathematics, Gaziantep, 27260, Turkey \\ In this paper, we consider the Potts model with competing interactions on the Cayley tree of order three. \\ We give the Potts model on the Cayley tree and its recursion relation. We construct the Gibbs states corresponding \\ to the model by using Markov random field method. We calculate the critical curve, such that there is a phase \\ transition for the model. We show that there are phase transition of the model for some given parameters. \\ We extend the results obtained by Akin and Temir (Condensed Matt. Phys. 14, 23003 (2011)).
}

DOI: 10.12693/APhysPolA.129.845

PACS/topics: 05.50.+q, 64.60.-i, 75.10.Hk, 64.60.De

\section{Introduction}

It is well known that lattice spin system is a large class of systems considered in statistical mechanics. Also it is well known that the structure of the lattice plays an important role in examinations of spin systems. The Potts model, which was introduced by Potts in 1952 as a generalization of the Ising model, has received an increasing theoretical and experimental aspects in recent years [1]. The idea came from the representation of the Ising model as interacting spins which can be either parallel or anti parallel [2]. One of important advantages of models on Bethe lattice is that no approximations have to be made and the calculations can be carried out with high accuracy. Studies of such model may lead to a discovery of more realistic systems with similar properties. The lattice spin system is a large class of systems considered in statistical mechanic [1]. The structure of the lattice also plays an important role in examining the spin systems. The Potts model has drawn an increased attention to its theoretical and experimental aspects in recent years [3]. It is well known that lattice spin system is a large class of systems considered in statistical mechanics. It is also well known that the structure of the lattice plays an important role in examining the spin systems. The Potts model, as a generalization of the Ising model on Cayley tree with competing interactions appeared in a pioneering work of Vannimenus [4], has recently been studied extensively (see $[1,3,5,6]$ ). On the Cayley tree one can consider two types of next-nearest-neighbors (triple neighbors): prolonged and one-level next-nearest-neighbors (respectively two-level triple neighbors).

For many problems the solution on a tree is much simpler than on a regular lattice and is equivalent to the

*corresponding author; e-mail: hasan.akin@zirve.edu.tr standard Bethe-Peierls theory [2]. In the recent years, many researchers have studied the existence of Gibbs measures corresponding to the Ising and Potts models on the Cayley tree.

The approach used to study Gibbs measure on Cayley tree is based on the Markov random field on trees and recurrent equations of this theory [2-5]. In [4], we have analytically studied the recurrence equations of a generalized (Axial Next-Nearest-Neighbor Ising) ANNNI model on a Cayley tree and obtained some exact results: critical temperatures and curves, number of phases, partition function.

In this paper, we consider the Potts model with three competing interactions and nonzero external field on the Bethe lattice of order three. We construct the Gibbs states corresponding to the model by means of Markov random field method. We calculate the critical curve such that there is a phase transition for the model. We extend the results obtained by Akin and Temir [1]. By using elementary calculations, we show that for some parameters $\theta, \theta_{1}, \theta_{2}$ and $\theta_{3}$, corresponding to the coupling constants given in the Hamiltonian, there are phase transitions.

\section{The model and recursive equations for partition function}

In this section, we are going to obtain recursive equations for partition function to examine Gibbs measures of the model.

Now, let us give some definitions to construct the model.

A Cayley tree $\Gamma^{k}$ of order $k \geq 1$ is an infinite tree, i.e., a graph without cycles with exactly $k+1$ edges issuing from each vertex. Cayley tree is denoted by $\Gamma^{k}=(V, \Lambda)$, where $V$ is the set of vertices of $\Gamma^{k}, \Lambda$ is the set of edges of $\Gamma^{k}$. Two vertices $x$ and $y, x, y \in V$ are called nearest-neighbors if there exists an edge $l \in \Lambda$ connecting them, which is denoted by $l=\langle x, y\rangle$. The distance $d(x, y), x, y \in V$, on the Cayley tree $\Gamma^{k}$, is the 
number of edges in the shortest path from $x$ to $y$. For a fixed vertex $x^{0} \in V$ we set $W_{n}=\left\{x \in V \mid d\left(x, x^{0}\right)=n\right\}$, $V_{n}=\left\{x \in V \mid d\left(x, x^{0}\right) \leq n\right\}$ and $L_{n}$ denotes the set of edges in $V_{n}$. The fixed vertex $x^{0}$ is called the zero-th level and the vertices in $W_{n}$ are called the $n$-th level. For the sake of simplicity we put $|x|=d\left(x, x^{0}\right), x \in V$. Two vertices $x, y \in V$ are called one level next nearest neighbors if $d(x, y)=2$. The next nearest neighbor vertices $x$ and $y$ are called one level next-nearest-neighbors if $|x| \neq|y|,(>x, y<)$.

The (formal) Hamiltonian of Potts model is

$$
\begin{gathered}
H(\sigma)=-J \sum_{\langle x, y\rangle} \delta_{\sigma(x) \sigma(y)}-J_{1} \sum_{\langle x, y\langle} \delta_{\sigma(x) \sigma(y)} \\
-J_{2} \sum_{\langle x, y, z\rangle} \delta_{\sigma(x) \sigma(y) \sigma(z)}-h \sum_{x \in V} \delta_{1 \sigma(x)},
\end{gathered}
$$

where the first sum ranges over all nearest neighbors, the second sum ranges over all the second neighbors, the third sum ranges over all triples of neighbors.

We assume the following Kronecker's Delta function for the triple neighbors

$$
\delta_{\sigma(x) \sigma(y) \sigma(z)}= \begin{cases}1, & \sigma(x)=\sigma(y)=\sigma(z), \\ 1 / 2, & \sigma(x)=\sigma(y) \neq \sigma(z) \\ & \text { or } \sigma(x) \neq \sigma(y)=\sigma(z), \\ 0, & \text { otherwise, }\end{cases}
$$

where $x, y \in W_{n}$ and $y \in W_{n-1}$ for all $n$ and we can also express (2) as $\delta_{\sigma(x) \sigma(y) \sigma(z)}=\frac{1}{2}\left(\delta_{\sigma(x) \sigma(y)}+\delta_{\sigma(y) \sigma(z)}\right)$.

The spin variables $\sigma(x)$ have their values on a set $\Phi=\{1,2, \ldots, q\}, q>2$ (we consider $q=3$ ), then also $J, J_{1}, J_{2} \in \mathrm{R}$ are the coupling constants and $h$ is the external field. The exact solutions of the Potts model with $J_{1}=0$ and $h=0$ has been studied [2]. In our model, we have chosen that all parameters $J, J_{1}, J_{2}$ and $h$ are nonzero.

Let $\Lambda$ be a finite subset of $V$. We will express the restriction of $\sigma$ to $\Lambda$ with $\sigma(\Lambda)$ and $\bar{\sigma}(V / \Lambda)$ will be a fixed boundary condition of spin variables. The full energy of $\sigma(\Lambda)$ with determined configuration of $\bar{\sigma}(V / \Lambda)$ is obtained by the following Hamiltonian:

$$
\begin{gathered}
H_{\Lambda}(\sigma(\Lambda) \mid \bar{\sigma}(V / \Lambda))=-J \sum_{\substack{\langle x, y\rangle \\
x, y \in \Lambda}} \delta_{\sigma(x) \sigma(y)}-J \sum_{\substack{\langle x, y\rangle \\
x \in \Lambda, y \notin \Lambda}} \delta_{\sigma(x) \sigma(y)} \\
-J_{1} \sum_{\substack{>x, y\langle \\
x, y \in \Lambda}} \delta_{\sigma(x) \sigma(y)}-J_{1} \sum_{\substack{\lambda x, y\langle \\
x \in \Lambda, y \notin \Lambda}} \delta_{\sigma(x) \sigma(y)}-J_{2} \sum_{\substack{\langle x, y, z\rangle \\
x, z, y \in \Lambda}} \delta_{\sigma(x) \sigma(y) \sigma(z)} \\
-J_{2} \sum_{\substack{\langle x, y, z\rangle \\
x, z \notin \Lambda, y \in \Lambda}} \delta_{\sigma(x) \sigma(y) \sigma(z)}-h \sum_{x \in \Lambda} \delta_{1 \sigma(x)},
\end{gathered}
$$

where $\delta_{\sigma(x) \sigma(y)}$ is the Kronecker's Delta function. Afterward the partition function $Z_{\Lambda}(\bar{\sigma}(V / \Lambda))$, which determines the boundary condition $\bar{\sigma}(V / \Lambda)$ in part of $\Lambda$ is defined as follows

$$
Z_{\Lambda}(\bar{\sigma}(V / \Lambda))=\sum_{\sigma(\Lambda) \in \Omega(\Lambda)} \exp \left[-\beta H_{\Lambda}(\sigma(\Lambda) \mid \bar{\sigma}(V / \Lambda))\right]
$$

where $\beta=1 / k T$ is the inverse temperature and $\Omega(\Lambda)$ is the set of all possibility of $\Lambda$. We introduce new notations $\sigma_{n}$ and $Z^{(n)}$ in place of configurations $\sigma\left(V_{n}\right)$ and the partition functions $Z_{V_{n}}$ respectively, inside the volume $V_{n}[2]$. The Gibbs measure is a probability measure, frequently seen in many problems of probability theory and statistical mechanics [7]. The Gibbs measure for a given configuration $\sigma_{n}$ is defined by

$$
\mu\left(\sigma_{\Lambda}\right)=\left(Z_{\Lambda}(\sigma)\right)^{-1} \exp \left[-\beta H\left(\sigma_{\Lambda}\right)\right],
$$

where $Z_{\Lambda}(\sigma)=\sum_{\sigma_{\Lambda} \in \Omega_{V}} \exp \left[-\beta H\left(\sigma_{\Lambda}\right)\right]$ is the partition function. We can normally define the concept of the Gibbs measure (phase) of the Potts model with three types of interactions on the Cayley tree of order 3 . Now, let us express the partition function $Z^{(n)}$ with a summation of the partial partition functions $Z_{i}^{(n)}$, as $Z^{(n)}=\sum_{i=1}^{q} Z_{i}^{(n)}$, where

$$
Z_{i}^{(n)}=\sum_{\sigma_{n} \in \Omega\left(V_{n}\right): \sigma\left(x^{0}\right)=i} \exp \left[-\beta H_{V_{n}}\left(\sigma_{n} \mid \bar{\sigma}\left(V / V_{n}\right)\right)\right] .
$$

The partition function is recurrently obtained, by beginning from the boundary conditions and continuing to the inner points of the Cayley tree of order three. The partial partition function of a branch on $n$ generations, where the innermost site is in state $i$, is denoted by $Z_{i}^{(n)}$. Given a fixed point (root of tree) $x^{0}$ and $x^{1}, x^{2}, x^{3} \in W_{1}$, let us denote $S\left(x^{0}\right)=\left\{x^{1}, x^{2}, x^{3}\right\}, \sigma\left(x^{0}\right)=i, \sigma\left(x^{1}\right)=j, \sigma\left(x^{2}\right)=$ $m, \sigma\left(x^{3}\right)=k$. Now we can define the partial partition functions of the following generations, for a given $n$, by $Z_{i}^{(n+1)}=\sum_{j, m, k=1}^{3} \exp \left[\beta\left(J\left(\delta_{i j}+\delta_{i k}+\delta_{i m}\right)+J_{1}\left(\delta_{j k}+\right.\right.\right.$ $\left.\left.\left.\delta_{j m}+\delta_{k m}\right)+J_{2}\left(\delta_{j i m}+\delta_{k i m}+\delta_{j i k}\right)+h \delta_{1 i}\right)\right] Z_{j}^{(n)} Z_{m}^{(n)} Z_{k}^{(n)}$, where $i=1,2,3$. Let $\theta=\exp [\beta J], \theta_{1}=\exp \left[\beta J_{1}\right]$, $\theta_{2}=\exp \left[\beta J_{2}\right], \theta_{3}=\exp [\beta h]$ for brevity.

Then the partial partition function can be obtained using (3) and (4) as follows:

$$
\begin{aligned}
& Z_{1}^{(n+1)}=\theta_{3}\left(\theta_{1}^{3}\left(\theta^{3} \theta_{2}^{3}\left(Z_{1}^{(n)}\right)^{3}+\left(Z_{2}^{(n)}\right)^{3}+\left(Z_{3}^{(n)}\right)^{3}\right)\right. \\
& \quad+6 \theta \theta_{2}\left(Z_{1}^{(n)} Z_{2}^{(n)} Z_{3}^{(n)}\right)+3 \theta^{2} \theta_{1} \theta_{2}^{2}\left(Z_{1}^{(n)}\right)^{2} \\
& \quad \times\left(Z_{2}^{(n)}+Z_{3}^{(n)}\right)+3 \theta \theta_{1} \theta_{2} Z_{1}^{(n)}\left(\left(Z_{2}^{(n)}\right)^{2}+\left(Z_{3}^{(n)}\right)^{2}\right) \\
& \left.\quad+3 \theta_{1} Z_{2}^{(n)} Z_{3}^{(n)}\left(Z_{2}^{(n)}+Z_{3}^{(n)}\right)\right) \\
& \quad Z_{2}^{(n+1)}=\left(\theta_{1}^{3}\left(\left(Z_{1}^{(n)}\right)^{3}+\theta^{3} \theta_{2}^{3}\left(Z_{2}^{(n)}\right)^{3}+\left(Z_{3}^{(n)}\right)^{3}\right)\right. \\
& \quad+6 \theta \theta_{2}\left(Z_{1}^{(n)} Z_{2}^{(n)} Z_{3}^{(n)}\right)+3 \theta \theta_{1} \theta_{2} Z_{2}^{(n)} \\
& \quad \times\left(\left(Z_{1}^{(n)}\right)^{2}+\left(Z_{3}^{(n)}\right)^{2}\right)+3 \theta^{2} \theta_{1} \theta_{2}^{2}\left(Z_{2}^{(n)}\right)^{2} \\
& \left.\quad \times\left(Z_{1}^{(n)}+Z_{3}^{(n)}\right)+3 \theta_{1} Z_{1}^{(n)} Z_{3}^{(n)}\left(Z_{1}^{(n)}+Z_{3}^{(n)}\right)\right) \\
& Z_{3}^{(n+1)}=\left(\theta_{1}^{3}\left(\left(Z_{1}^{(n)}\right)^{3}+\left(Z_{2}^{(n)}\right)^{3}+\theta^{3} \theta_{2}^{3}\left(Z_{3}^{(n)}\right)^{3}\right)\right. \\
& \quad+6 \theta \theta_{2}\left(Z_{1}^{(n)} Z_{2}^{(n)} Z_{3}^{(n)}\right)+3 \theta \theta_{1} \theta_{2} Z_{3}^{(n)}
\end{aligned}
$$




$$
\begin{aligned}
& \times\left(\left(Z_{1}^{(n)}\right)^{2}+\left(Z_{2}^{(n)}\right)^{2}\right)+3 \theta^{2} \theta_{1} \theta_{2}^{2}\left(Z_{3}^{(n)}\right)^{2} \\
& \left.\times\left(Z_{1}^{(n)}+Z_{2}^{(n)}\right)+3 \theta_{1} Z_{1}^{(n)} Z_{2}^{(n)}\left(Z_{1}^{(n)}+Z_{2}^{(n)}\right)\right)
\end{aligned}
$$

We have the following recurrent system of equations, after substitution $u_{n+1}=Z_{2}^{(n+1)} / Z_{1}^{(n+1)}$ and $v_{n+1}=$ $Z_{3}^{(n+1)} / Z_{1}^{(n+1)}$

$$
\begin{aligned}
& \theta_{3} u_{n+1}=\frac{\theta_{1}^{3}\left(1+\theta^{3} \theta_{2}^{3} u_{n}^{3}+v_{n}^{3}\right)+6 \theta \theta_{2} u_{n} v_{n}+3 \theta \theta_{1} \theta_{2} u_{n}\left(1+v_{n}^{2}\right)+3 \theta_{1} v_{n}\left(1+v_{n}\right)+3 \theta^{2} \theta_{1} \theta_{2}^{2} u_{n}^{2}\left(1+v_{n}\right)}{\theta_{1}^{3}\left(\theta^{3} \theta_{2}^{3}+u_{n}^{3}+v_{n}^{3}\right)+6 \theta \theta_{2} u_{n} v_{n}+3 \theta^{2} \theta_{1} \theta_{2}^{2}\left(u_{n}+v_{n}\right)+3 \theta \theta_{1} \theta_{2}\left(u_{n}^{2}+v_{n}^{2}\right)+3 \theta_{1} u_{n} v_{n}\left(v_{n}+u_{n}\right)} \\
& \theta_{3} v_{n+1}=\frac{\theta_{1}^{3}\left(1+u_{n}^{3}+\theta^{3} \theta_{2}^{3} v_{n}^{3}\right)+6 \theta \theta_{2} u_{n} v_{n}+3 \theta_{1} u_{n}\left(1+u_{n}\right)+3 \theta \theta_{1} \theta_{2} v_{n}\left(1+u_{n}^{2}\right)+3 \theta^{2} \theta_{1} \theta_{2}^{2} v_{n}^{2}\left(1+u_{n}\right)}{\theta_{1}^{3}\left(\theta^{3} \theta_{2}^{3}+u_{n}^{3}+v_{n}^{3}\right)+6 \theta \theta_{2} u_{n} v_{n}+3 \theta^{2} \theta_{1} \theta_{2}^{2}\left(u_{n}+v_{n}\right)+3 \theta \theta_{1} \theta_{2}\left(u_{n}^{2}+v_{n}^{2}\right)+3 \theta_{1} u_{n} v_{n}\left(u_{n}+v_{n}\right)} .
\end{aligned}
$$

We describe a function $F=\left(F_{1}, F_{2}\right): \mathrm{R}^{2} \rightarrow \mathrm{R}^{2}$ with $u_{n+1}=F_{1}\left(u_{n}, v_{n}\right)$ and $v_{n+1}=F_{2}\left(u_{n}, v_{n}\right)$. We are going to try to get solutions, called fixed points, of the function $w=F(w)$, where $w=\left(u_{n}, v_{n}\right)$. Existence of the translation-invariant Gibbs measures (phases) of the Potts model produced by the Hamiltonian (1) is dependent on the number of the roots of the function $w=F(w)$. The recursive equations (6) can be written as $w_{n}=F\left(w_{n-1}\right), n>0$. In the theory of dynamical systems, $w_{n}$ is called trajectory of the initial point $w_{1}$ under the action of the mapping $F$. So, asymptotic behavior of $Z^{(n)}$ for $n \rightarrow \infty$ can be determined by value of $\lim _{n} w_{n}$. After replacement $u=\lim _{n \rightarrow \infty} u_{n}$ and $v=\lim _{n \rightarrow \infty} v_{n}$, we get the following equations:

$$
\begin{aligned}
& \theta_{3} u=\frac{\theta_{1}^{3}+\theta^{3} \theta_{1}^{3} \theta_{2}^{3} u^{3}+\theta_{1}^{3} v^{3}+6 \theta_{2} u v+3 \theta_{1} \theta_{2} u+3 \theta_{1} v+3 \theta^{2} \theta_{1} \theta_{2}^{2} u^{2}+3 \theta^{2} \theta_{1} \theta_{2}^{2} u^{2} v+3 \theta_{1} v^{2}+3 \theta \theta_{1} \theta_{2} u v^{2}}{\theta^{3} \theta_{1}^{3} \theta_{2}^{3}+\theta_{1}^{3} u^{3}+\theta_{1}^{3} v^{3}+6 \theta \theta_{2} u v+3 \theta^{2} \theta_{1} \theta_{2}^{2} u+3 \theta^{2} \theta_{1} \theta_{2}^{2} v+3 \theta_{1} \theta_{2} u^{2}+3 \theta_{1} u^{2} v+3 \theta \theta_{1} \theta_{2} v^{2}+3 \theta_{1} u v^{2}}, \\
& \theta_{3} v=\frac{\theta_{1}^{3}+\theta_{1}^{3} u^{3}+\theta^{3} \theta_{1}^{3} \theta_{2}^{3} v^{3}+6 \theta \theta_{2} u v+3 \theta_{1} u+3 \theta \theta_{1} \theta_{2} v+3 \theta_{1} u^{2}+3 \theta_{1} \theta_{2} u^{2} v+3 \theta^{2} \theta_{1} \theta_{2}^{2} v^{2}+3 \theta^{2} \theta_{1} \theta_{2}^{2} u v^{2}}{\theta^{3} \theta_{1}^{3} \theta_{2}^{3}+\theta_{1}^{3} u^{3}+\theta_{1}^{3} v^{3}+6 \theta \theta_{2} u v+3 \theta^{2} \theta_{1} \theta_{2}^{2} u+3 \theta^{2} \theta_{1} \theta_{2}^{2} v+3 \theta \theta_{1} \theta_{2} u^{2}+3 \theta_{1} u^{2} v+3 \theta \theta_{1} \theta_{2} v^{2}+3 \theta_{1} u v^{2}} .
\end{aligned}
$$

After some simplification, equations (7) can be written as follows:

$$
\begin{aligned}
& \theta_{3} u=\frac{\theta_{1}^{3}\left(\theta^{3} \theta_{2}^{3} u^{3}+\left(v^{3}+1\right)\right)+3 \theta \theta_{2}\left(2 u v+\theta_{1} u\left(v^{2}+1\right)\right)+\left(3 \theta_{1} v+3 \theta^{2} \theta_{1} \theta_{2}^{2} u^{2}\right)(v+1)}{\theta_{1}^{3}\left(u^{3}+v^{3}\right)+3 \theta \theta_{2}\left(\theta_{1}\left(u^{2}+v^{2}\right)+2 u v\right)+3 \theta_{1}\left(\theta^{2} \theta_{2}^{2}+u v\right)(u+v)+\theta^{3} \theta_{1}^{3} \theta_{2}^{3}} \\
& \theta_{3} v=\frac{\theta_{1}^{3}\left(\left(1+u^{3}\right)+\theta^{3} \theta_{2}^{3} v^{3}\right)+6 \theta \theta_{2} u v+3 \theta \theta_{1} \theta_{2} v\left(1+u^{2}\right)+3 \theta_{1}(1+u)\left(u+\theta^{2} \theta_{2}^{2} v^{2}\right)}{\theta_{1}^{3}\left(u^{3}+u^{3}\right)+3 \theta \theta_{1} \theta_{2}\left(u^{2}+u^{2}\right)+6 \theta \theta_{2} u v+3 \theta_{1}(u+v)\left(\theta^{2} \theta_{2}^{2}+u v\right)+\theta^{3} \theta_{1}^{3} \theta_{2}^{3}} .
\end{aligned}
$$

\section{Translation-invariant Gibbs measures}

If there exists more than one Gibbs state for a given potential $V$, we can say that the phase transition occurs [2]. We can mention about a phase transition of a nonlinear dynamical system without a unique Gibbs state. In this part of paper we will solve the equation system (7) to find the phase transition. Assuming that $u=v$ for (7) we then obtain the following equation

$$
u=\frac{\theta_{1}\left(\theta_{1}^{2}+\theta^{3} \theta_{1}^{2} \theta_{2}^{3}+3 \theta \theta_{2}+3 \theta^{2} \theta_{2}^{2}\right) u^{3}+3\left(2 \theta \theta_{2}+\theta_{1}+\theta^{2} \theta_{1} \theta_{2}^{2}\right) u^{2}+\left(3 \theta_{1} \theta_{2}+3 \theta\right) u+\theta_{1}^{3}}{\theta_{3}\left(\left(2 \theta_{1}^{3}+6 \theta_{1}\right) u^{3}+\left(6 \theta \theta_{2}+6 \theta \theta_{1} \theta_{2}\right) u^{2}+\left(6 \theta^{2} \theta_{1} \theta_{2}^{2}\right) u+\theta^{3} \theta_{1}^{3} \theta_{2}^{3}\right)} .
$$

We can show the last Eq. (8) as $u=f(u)$. The solutions of the system (8) describe the translation-invariant Gibbs measures corresponding to model (1). The number of the solutions of the Eq. (8) depends on the parameters $\beta=\frac{1}{k T}, \theta, \theta_{1}, \theta_{2}, \theta_{3}$ and $h$. The phase transition usually occurs at low temperature. It is possible to find an exact value of $T^{*}$ such that a phase transition occurs for all
$T<T^{*}$ where $T^{*}$ is called a critical value of temperature. After some notations and simple calculations in Eq. 8, we can get the following fourth order equation,

$$
\begin{aligned}
& g(u)=D u^{4}+(E-A) u^{3}+(F-B) u^{2} \\
& \quad+(G-C) u+H=0,
\end{aligned}
$$


where

$$
\begin{array}{ll}
A=\theta_{1}^{3}\left(1+\theta^{3} \theta_{2}^{3} 9+3 \theta \theta_{1} \theta_{2}\left(1+\theta \theta_{2}\right),\right. & E=6 \theta \theta_{2} \theta_{3}\left(1+\theta_{1}\right), \\
B=3 \theta \theta_{2}\left(2+\theta \theta_{1} \theta_{2}\right)+3 \theta_{1}, & F=6 \theta^{2} \theta_{1} \theta_{2}^{2} \theta_{3}, \\
C=3 \theta_{1}\left(\theta \theta_{2}+1\right), & G=\theta^{3} \theta_{1}^{3} \theta_{2}^{3}, \\
D=2 \theta_{1}^{3} \theta_{3}+6 \theta_{1} \theta_{3}, & H=\theta_{1}^{3} .
\end{array}
$$

Now we will analyze how many roots (positive, negative or complex) has Eq. (9). Then we will look for the conditions for which Eq. (9) has the maximum possible number of positive roots by using the Descartes' Rule of Signs. Thus, we consider all probabilities of the other coefficient's sign to determine the maximum number of positive real roots (or zeroes), which show the existence of the Gibbs measures, ( $u$-intercepts) for the polynomial (9). The following table summarizes signs of coefficients of the polynomial to determine conditions at which the equation (9) has the maximum possible number of positive roots.

TABLE I

Number of all possible roots (positive, negative and complex) of the Eq. (9).

\begin{tabular}{c|c|c|c|c|c}
\hline \hline E-A & F-B & G-C & Positive & Negative & Complex \\
\hline+ & + & + & 0 & $4,2,0$ & $0,2,4$ \\
\hline+ & + & - & 2 & 2,0 & 0,2 \\
\hline+ & - & + & 2 & 2,0 & 0,2 \\
\hline+ & - & - & 2 & 2,0 & 0,2 \\
\hline- & + & + & 2 & 2,0 & 0,2 \\
\hline- & + & - & 4 & 0 & 0 \\
\hline- & - & + & 2 & 2,0 & 0,2 \\
\hline- & - & - & 2 & 0 & 2
\end{tabular}

According to Table I, Eq. (9) has the maximum number of possible positive roots (4) when $(E-A)<0,(F-B)>$ 0 and $(G-C)<0$ and the minimum number of positive roots $(0)$ when $(E-A)>0,(F-B)>0$ and $(G-C)>0$ (see Table I). Due to some parameters such as $\theta, \theta_{1}, \theta_{2}$ and $\theta_{3}$ the number of positive roots of the Eq. (8) changes normally. So we manipulate the equation to see where the Gibbs states occur.

Now we will approximately demonstrate some positive roots of the Eq. (8) on a graph by using Mathematica software. Such equations can be solved using known formulas, since we will have some complicated formulas for the coefficients and the solutions; we do not present the solution here. But, we have manipulated the Eq. (8) via Mathematica.

As shown in Fig. 1a the phase transition does not exist for $\theta \cong 0.001, \theta_{1} \cong 0.00001, \theta_{2} \cong 0.001$ and $\theta_{3} \cong 0.005$ because we have only one positive root. When we take the parameters as $\theta \cong 1.18, \theta_{1} \cong 0.86, \theta_{2} \cong 17.1$ and $\theta_{3} \cong$ 13.57 we get exactly two positive roots, shown in Fig. 1c. When we look at Fig. 1b, we have finally three roots where the parameters $\theta \cong 0.86, \theta_{1} \cong 0.94, \theta_{2} \cong 20.6$ and $\theta_{3} \cong 10.37$. Thus model (1) has a phase transition for cases (b) and (c) [2].

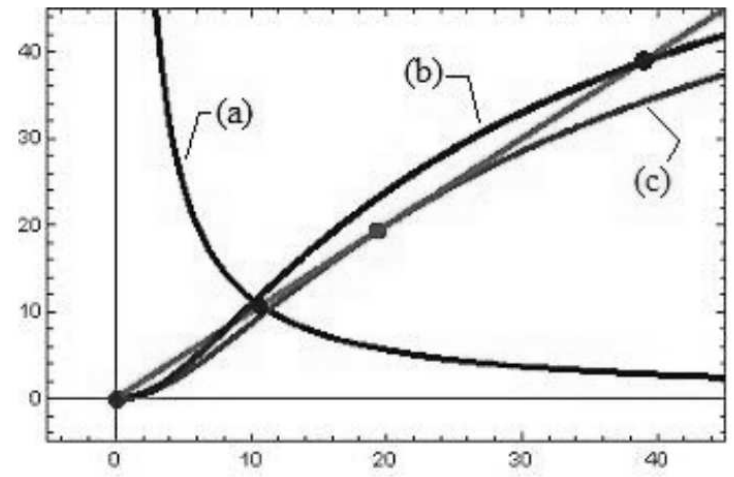

Fig. 1. Plot of the function $f(u)(8)$ for some values of the parameters $\theta, \theta_{1}, \theta_{2}$ and $\theta_{3}$.

\section{Conclusions}

In this paper we have obtained the exact solution of a phase transition problem by means of Gibbs state of the Potts model on a Bethe lattice of order three with three coupling constants $J, J_{1}, J_{2}$ and the external magnetic field constant $h$. Here we have extended studies from [2] by reproducing branch of Cayley tree from 2 to 3 . Hence, we have also seen the influence of branch number of the Bethe lattice $\left(\Gamma^{k}, k=3\right)$ on the existence of the phase transitions. In the future work, we are going to search whether the Gibbs states and phase transitions occur when different models are considered, such as mixed type Potts $\lambda$-model on Cayley tree of order three or more.

\section{References}

[1] F.Y. Wu, Rev. Mod. Phys. 54, 235 (1982).

[2] H. Akın, S. Temir, Cond. Matt. Phys. 14, 23003 (2011).

[3] U.A. Rozikov, H. Akın, S. Uguz, Math. Phys. Anal. Geom. 17, 103 (2014).

[4] H. Akın, U.A. Rozikov, S. Temir, J. Stat. Phys. 142, 314 (2011).

[5] H. Akın, N. Ganikhodjaev, S. Temir, S. Uguz, Acta Phys. Pol. A 123, 484 (2013).

[6] N. Ganikhodjaev, H. Akın, S. Uguz, S. Temir, J. Stat. Mech-Theory Exp. 2011, P03025 (2011).

[7] H.O. Georgii, Gibbs measures and phase transitions, De Gruyter studies in Mathematics Vol. 9, Berlin 1988. 